\title{
Inhibiting Glycosphingolipid Synthesis Ameliorates Hepatic Steatosis in Obese Mice
}

\author{
Hongmei Zhao, ${ }^{1}$ Malgorzata Przybylska,,${ }^{1}$ I-Huan Wu, ${ }^{1}$ Jinhua Zhang, ${ }^{1}$ Panagiotis Maniatis, ${ }^{1}$ Joshua Pacheco, ${ }^{1}$ \\ Peter Piepenhagen, ${ }^{1}$ Diane Copeland, ${ }^{1}$ Cynthia Arbeeny, ${ }^{1}$ James A. Shayman, ${ }^{2}$ Johannes M. Aerts, ${ }^{3}$ Canwen Jiang, ${ }^{1}$ \\ Seng H. Cheng, ${ }^{1}$ and Nelson S. Yew ${ }^{1}$
}

\begin{abstract}
Steatosis in the liver is a common feature of obesity and type 2 diabetes and the precursor to the development of nonalcoholic steatohepatitis (NASH), cirrhosis, and liver failure. It has been shown previously that inhibiting glycosphingolipid (GSL) synthesis increases insulin sensitivity and lowers glucose levels in diabetic rodent models. Here we demonstrate that inhibiting GSL synthesis in ob/ob mice not only improved glucose homeostasis but also markedly reduced the development of hepatic steatosis. The ob/ob mice were treated for 7 weeks with a specific inhibitor of glucosylceramide synthase, the initial enzyme involved in the synthesis of GSLs. Besides lowering glucose and hemoglobin A1c (HbA1c) levels, drug treatment also significantly reduced the liver/body weight ratio, decreased the accumulation of triglycerides, and improved several markers of liver pathology. Drug treatment reduced liver glucosylceramide (GL1) levels in the ob/ob mouse. Treatment also reduced the expression of several genes associated with hepatic steatosis, including those involved in lipogenesis, gluconeogenesis, and inflammation. In addition, inhibiting GSL synthesis in dietinduced obese mice both prevented the development of steatosis and partially reversed preexisting steatosis. Conclusion: These data indicate that inhibiting GSL synthesis ameliorates the liver pathology associated with obesity and diabetes, and may represent a novel strategy for treating fatty liver disease and NASH. (HерATOLOGY 2009;50:85-93.)
\end{abstract}

$\mathrm{N}$ onalcoholic fatty liver disease (NAFLD) is increasingly being recognized as a major health care problem, paralleling the profound increase in obesity and type 2 diabetes. ${ }^{1,2}$ The prevalence of hepatic steatosis in the U.S. is estimated to be $\approx 20 \%-30 \%$

\footnotetext{
Abbreviations: $A L T$, alanine aminotransferase; ChREBP, carbohydrate responsive element-binding protein; $C P T$, carnitine palmitoyltransferase; DIO, diet-induced obese; FFA, free fatty acid; GL1, glucosylceramide; GL2, lactosylceramide; GSL, glycosphingolipid; IR, insulin resistance; NAFLD, nonalcoholic fatty liver disease; NASH, nonalcoholic steatohepatitis; PPAR- $\alpha / \gamma$, peroxisome proliferatoractivated receptor $\alpha / \gamma ; S R E B P$, sterol regulatory element binding protein; TNF- $\alpha$, tumor necrosis factor- $\alpha$.

From ${ }^{1}$ Genzyme Corp., Framingham, MA; ${ }^{2}$ Nephrology Division, Department of Internal Medicine, University of Michigan, Ann Arbor, MI; ${ }^{3}$ Department of Medical Biochemistry, Academic Medical Center, Amsterdam, The Netherlands. Received December 14, 2008; accepted March 7, 2009.

Address reprint requests to: Seng H. Cheng, Ph.D., Genzyme Corp., 49 New York Avenue, Framingham, MA 01701-9322. E-mail: seng.cheng@genzyme; fax: 508-661-8812.

Copyright (C) 2009 by the American Association for the Study of Liver Diseases. Published online in Wiley InterScience (www.interscience.wiley.com). DOI 10.1002/hep.22970

Potential conflict of interest: Drs. Zhao, Przybylska, Wu, Zhang, Maniatis, Pacheco, Piepenhagen, Arbeeny, Jiang, Cheng, and Yew own stocks in Genzyme Corp. Dr. Shayman holds intellectual property rights for Genzyme.

Additional Supporting Information may be found in the online version of this article.
}

of the population, and hepatic steatosis is being detected in children with greater frequency. ${ }^{3,4}$ Given that persistent lipid accumulation in the liver can in some cases progress to the more serious conditions of nonalcoholic steatohepatitis (NASH) and cirrhosis, there is a considerable need for effective prophylactic and interventional therapies.

A series of metabolic abnormalities contribute to the development of hepatic steatosis. 5 Insulin resistance (IR) is closely associated with fatty liver disease and is a major consequence of obesity as well as a primary cause of steatosis in the liver. ${ }^{6}$ IR in adipocytes leads to increased activity of hormone-sensitive lipase and insufficient suppression of lipolysis in adipose tissue. These elevated free fatty acids (FFAs) flow to the serum and then to the liver. ${ }^{7}$ Hyperinsulinemia and hyperglycemia, also caused by IR, induce the expression of the key transcriptional regulators sterol regulatory elementbinding protein (SREBP)-1 and carbohydrate responsive element-binding protein (ChREBP), activating the genes involved in lipogenesis. In addition, the increased production of a key intermediate in the lipogenic pathway, malonyl-CoA, inhibits carnitine palmitoyltransferase (CPT)-1, which is required for 
FFA transport into the mitochondria. ${ }^{5}$ The combined influx of FFAs, increased de novo synthesis, and reduced FFA oxidation all serve to drive hepatic triglyceride production and lipid accumulation, leading to steatosis.

Although to date glycosphingolipids (GSLs) have not been considered as having a role in the development of hepatic steatosis, GSLs are known to be involved in the development of IR, based on studies in vitro and in ganglioside GM3 synthase knockout transgenic mice, which exhibit increased insulin sensitivity compared to wildtype mice and protection from a high-fat diet-induced IR. ${ }^{8-10}$ More recently, we have shown that inhibiting GSL synthesis improves glycemic control and increases insulin sensitivity in animal models of type 2 diabetes. ${ }^{11,12} \mathrm{Ad}-$ ministration of a small molecule inhibitor of glucosylceramide synthase (Genz-123346), which blocks the first step in the synthesis of several GSLs, lowered glucose levels and improved glucose tolerance in the Zucker diabetic fatty rat and in the diet-induced obese mouse. ${ }^{11,12}$ Treatment with a different inhibitor of glucosylceramide synthase (MZ-21) likewise lowered blood glucose and HbA1c levels, improved glucose tolerance, and increased insulin sensitivity in the muscle and liver of ob/ob mice. ${ }^{12}$ Given the close association between the development of IR and hepatic steatosis, these data support the prediction that improving insulin sensitivity by inhibiting GSL synthesis will also beneficially impact hepatic steatosis. In this study, we characterized the effects of Genz-123346 in the livers of ob/ob mice and diet-induced obese (DIO) mice. We observed improvements in reducing steatosis and markers of liver pathology as a result of drug treatment. In addition, we showed in DIO mice that inhibiting GSL synthesis not only prevented the development of hepatic lipid accumulation but also partially reversed established steatosis in older animals.

\section{Materials and Methods}

Animals. Male ob/ob mice (C57BL/6J-Lepob, Stock \#00063224), lean control mice, and male C57BL/6 mice were purchased from the Jackson Laboratories (Bar Harbor, ME). The lean controls were ob/? littermates, i.e., either heterozygous $(+/-)$ or wild-type $(+/+)$ with regard to the leptin gene. DIO mice were generated by placing male C57BL/6 mice, beginning at 5 weeks of age, on a high-fat diet (45\% kcal fat) for 8-31 weeks (D12451, Research Diets Inc., New Brunswick, NJ). Control animals were placed on a matched low-fat diet (10\% kcal fat) (D12450, Research Diets). The mice remained on either the high-fat or low-fat diet during the drug-treatment phase. Animals were cared for in an Association of Assess- ment and Accreditation of Laboratory Animal Care (AAALAC)-accredited facility in accordance with the guidelines established by the National Research Council.

Administration of Drug. The synthesis of Genz$123346\left[(1 R, 2 R)\right.$-nonanoic acid[2-( $\left(2^{\prime}, 3^{\prime}\right.$-dihydro-benzo $[1,4]$ dioxin-6'-yl)-2-hydroxy-1-pyrrolidin-1-ylmethylethyl]-amide-L-tartaric acid salt] is described in U.S. Patent No. 6,855,830. Animals were orally gavaged twice per day with a solution of Genz-123346 in water or water alone (control group). In some studies the drug was formulated in powdered food and placed in glass feeding jars. The amount of drug delivered was calculated from the amount of food eaten per mouse per day.

Blood Analysis. Blood samples were collected by either tail vein nick (glucose) or retroorbital plexus puncture (HbA1c). Nonfasting glucose levels were measured using an Accu-Chek Compact Meter (Roche Diagnostics, Indianapolis, IN). HbA1c levels were measured using A1cNow Monitors (Metrika Inc., Sunnyvale, CA). Alanine aminotransferase (ALT) levels in plasma were measured by IDEXX Laboratories (North Grafton, MA).

Measurement of Sphingolipid Levels. Ceramide and GSLs were analyzed using high-performance liquid chromatography / tandem mass spectrometry as described ${ }^{13}$ with some modifications (see Supporting Material). Liver protein concentration was determined by the RC DC Protein Assay Kit (Bio-Rad Laboratories, Hercules, CA).

Histology and Quantification of Steatosis. Livers were fixed in $10 \%$ neutral buffered formalin and then embedded in paraffin. Sections were then stained with hematoxylin-eosin. To visualize neutral lipids, livers were frozen in Tissue-Tek O.C.T. compound (Sakura Finetek USA Inc., Torrance, CA) and sections were stained with Oil Red O. Hepatic steatosis was quantitated by morphometric analysis (see Supporting Material).

Liver Fat Mass and Quantitation of Liver Triglycerides. Fat and lean masses of the livers were determined using a quantitative magnetic resonance body composition and tissue analyzer (EchoMRI 3-in-1, Echo Medical Systems, Houston, TX). To measure triglyceride levels, livers were homogenized in water and total lipids were extracted with methanol:chloroform (4:3.3). The extracted lipids were dried in glass vials and then resuspended in dimethylsulfoxide. Triglycerides were measured using the Serum Triglyceride Determination kit (TR0100, Sigma-Aldrich, St. Louis, MO).

RNA Expression Analysis by Quantitative ReverseTranscriptase Polymerase Chain Reaction (RT-PCR). Total RNA from liver was isolated using the RNeasy Lipid Tissue kit (Qiagen Inc., Valencia, CA). Quantitative RT-PCR was performed using the TaqMan OneStep RT-PCR Master Mix Reagents kit (Applied 
A
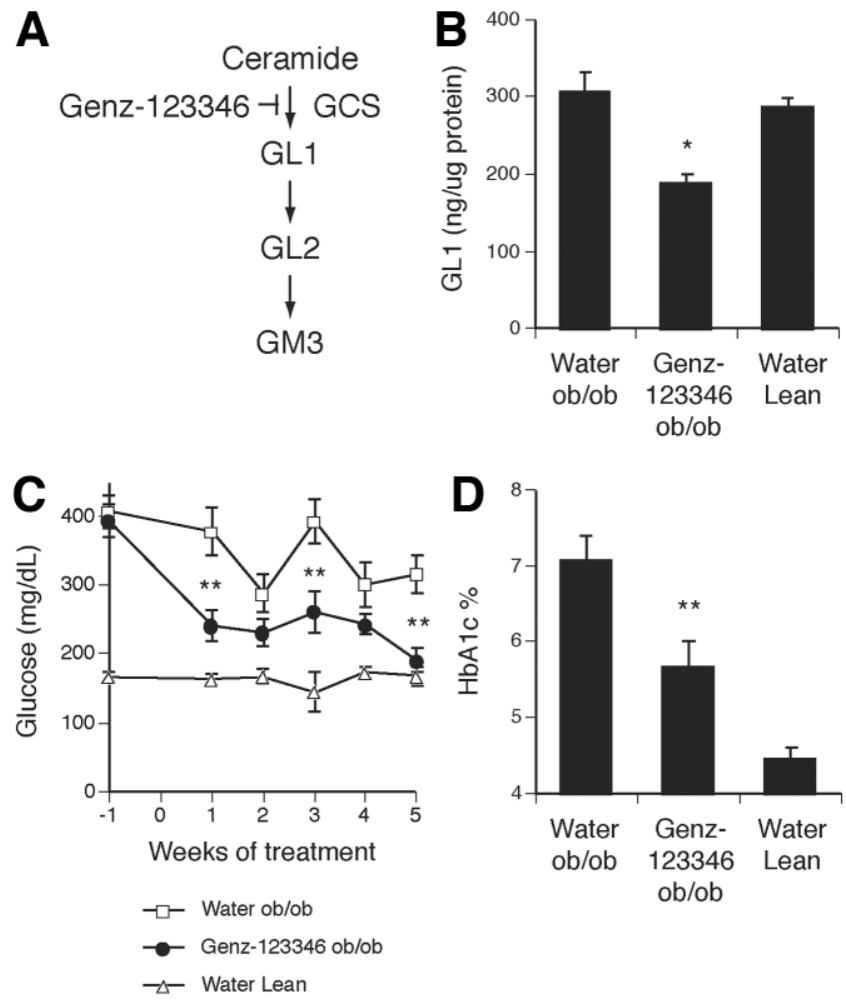

Fig. 1. Effect of Genz-123346 on liver GL1, blood glucose, and HbA1c levels in ob/ob mice. Beginning at 7 weeks of age, male mice were orally gavaged with Genz-123346 (60 mg/kg twice daily) or placebo (water) for 7 weeks. (A) Diagram of the initial step inhibited by Genz-123346. GCS, glucosylceramide synthase; GL1, glucosylceramide; GL2, lactosylceramide. (B) Liver GL1 levels at the end of the treatment period. (C) Nonfasting glucose levels from week -1 to week 5. (D) HbA1c levels (percent of total hemoglobin) after 6 weeks of treatment. Data shown as mean \pm standard error of the mean (SEM) ( $n=8$ mice per group). ${ }^{*} P<0.05,{ }^{*} * P<0.01 \mathrm{Genz}-123346 \mathrm{ob} / \mathrm{ob}$ versus water ob/ob.

Biosystems, Foster City, CA) and predesigned TaqMan Gene Expression Assays (Applied Biosystems) for each of the genes analyzed. Ribosomal 18S RNA was used as an internal standard.

Statistical Analysis. Data were analyzed by one-way analysis of variance (ANOVA) followed by Bonferroni's post-hoc test using the Prism 4 software program (GraphPad Software Inc., San Diego, CA). Data were considered significant if $P<0.05$.

\section{Results}

Effect of Genz-123346 on Liver GL1 Levels. Genz123346 is a highly specific inhibitor of glucosylceramide (GL1) synthase (Fig. 1A). ${ }^{11}$ The compound is rapidly metabolized in rodents (half-life $<20$ minutes in plasma), and requires daily or twice daily administration in order to lower GSLs. Genz-123346 was administered to 7-weekold ob/ob mice by daily or twice-daily oral gavage $(60$ $\mathrm{mg} / \mathrm{kg} /$ dose $=120 \mathrm{mg} / \mathrm{kg} /$ day) for 6-7 weeks. The drug was well tolerated with minimal effects on food consumption or body weight gain (data not shown). We quantitated the levels of GL1 from the livers of ob/ob mice and lean mice (ob/? littermates that were either heterozygous or wild-type with regard to the leptin gene) using highperformance liquid chromatography and tandem mass spectrometry. Liver GL1 levels in ob/ob mice were comparable to the levels in lean mice (Fig. 1B). Drug treatment reduced GL1 levels by $\approx 40 \%(P<0.05)$ compared to the placebo (water) treated animals. The levels of GM3 trended lower but were not significantly altered as a result of drug treatment, and the levels of ceramide and GL2 were unchanged (data not shown). Although the variability between animals and inherent in vivo instability of the compound limited observing more significant or greater changes in the levels of the various sphingolipids, administration of Genz-123346 decreased GL1 levels in the treated animals as expected.

Effect of Genz-123346 on Glucose and HbA1c Levels in ob/ob Mice. To determine if Genz-123346 improved glucose homeostasis in ob/ob mice, the drug was administered by twice-daily oral gavage $(60 \mathrm{mg} / \mathrm{kg} / \mathrm{dose})$ for 7 weeks. Nonfasting glucose levels in the water-treated control group varied between $300-400 \mathrm{mg} / \mathrm{dL}$ during the course of the study (Fig. 1C). Glucose levels in the group treated with Genz-123346 declined within the first week of treatment, and remained within the range of 200-250 $\mathrm{mg} / \mathrm{dL}$ for the next 4 weeks. Although we observed considerable day-to-day variability in nonfasting glucose levels, after 6 weeks of treatment we consistently observed a significant reduction in HbA1c levels in the animals treated with Genz-123346 (Fig. 1D), indicating an improvement in glucose homeostasis in the ob/ob mice as a result of drug treatment.

Genz-123346 Decreased Hepatic Steatosis in ob/ob Mice. We next characterized the effects of treatment with Genz-123346 on liver pathology. After 7 weeks of treatment, the livers were harvested and weighed. The ob/ob mice exhibited typically larger and heavier livers (as a percentage of body weight) compared to lean animals (Fig. 2A). Although drug treatment had a minimal effect on overall body weight gain compared to the placebo-treated group, animals treated with Genz-123346 exhibited decreased liver weights (Fig. 2A). Drug treatment also decreased the serum levels of the liver transaminase ALT (Fig. 2B), albeit not to normal levels.

The livers were subsequently sectioned and stained with hematoxylin and eosin or with Oil Red O, which stains neutral lipids. In the placebo (water)-treated ob/ob mice, numerous lipid-filled vacuoles and "foamy" cells were present throughout the parenchyma, which is characteristic of the extensive hepatic steatosis that develops in 

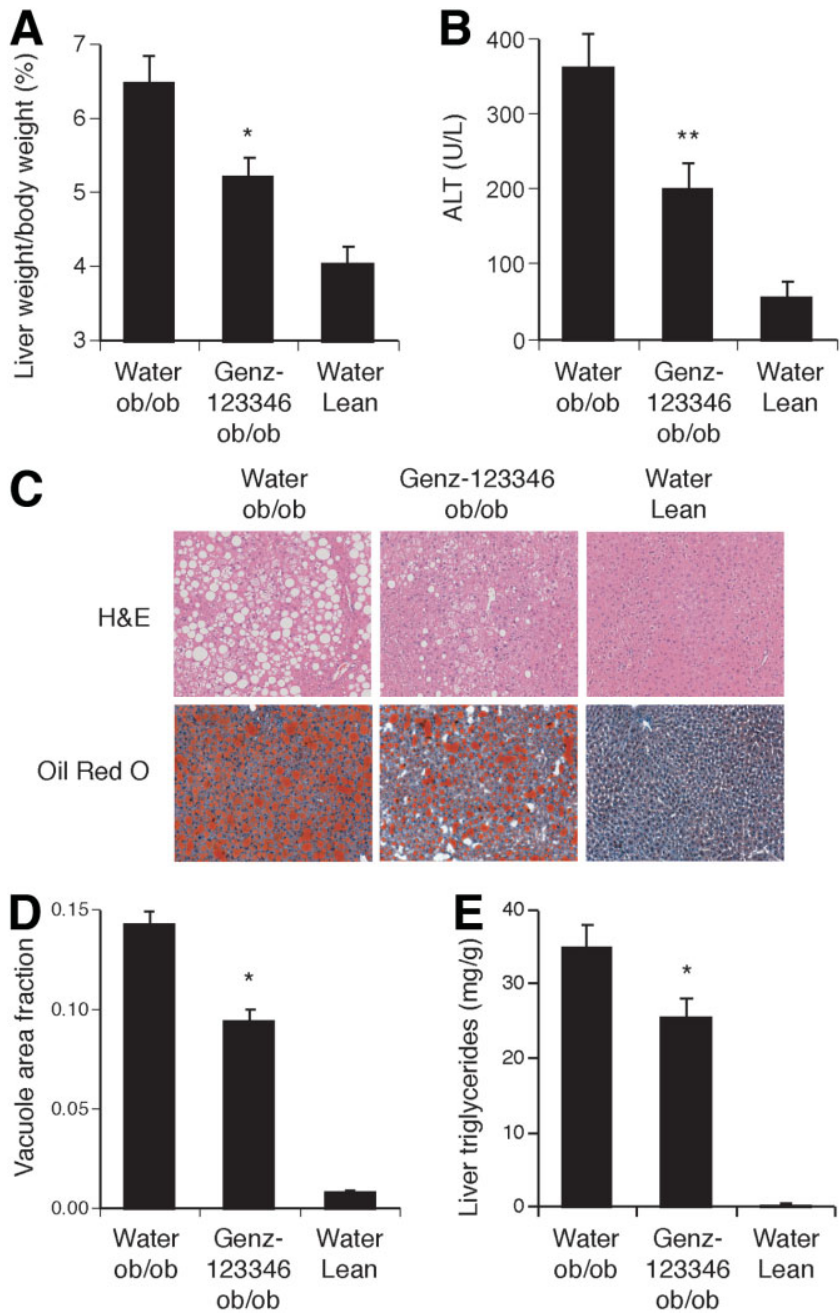

Fig. 2. Genz-123346 decreased liver weights, ALT levels, and hepatic steatosis in ob/ob mice. (A) After 7 weeks of treatment with Genz-123346 (60 mg/kg twice daily oral gavage $=120 \mathrm{mg} / \mathrm{kg} /$ day $)$ or placebo (water), livers were weighed and plotted as a percentage of total body weight. (B) Serum was collected from the mice after 7 weeks of treatment and the levels of ALT measured. (C) Representative liver sections stained with hematoxylin and eosin (H\&E) or Oil Red 0. (D) Fractional area of the livers containing vacuoles as determined by morphometric analysis (see Materials and Methods). (E) Total liver triglyceride levels. Data shown as mean \pm SEM $(n=$ 8 mice per group). ${ }^{*} P<0.05,{ }^{*} * P<0.01$ Genz-123346 ob/ob versus water ob/ob.

these mice (Fig. 2C). In the ob/ob mice treated with Genz-123346, the size and number of vacuoles and foamy cells were decreased relative to the placebo-treated control animals. This decrease was confirmed by morphometric analysis that quantitated the fractional area occupied by vacuoles for each liver section. There was a significant $(P<0.05)$ decrease in vacuole area fraction in the ob/ob mice treated with Genz-123346 (Fig. 2D). Total liver triglycerides were also determined, and reduced triglyceride levels were observed in the drug-treated ob/ob mice relative to the placebo-treated group (Fig. 2E). These results show that Genz-123346 improved the overall gross pathology of the liver and significantly decreased hepatic steatosis in $\mathrm{ob} / \mathrm{ob}$ mice.

Effect of Genz-123346 on the Expression of Genes Involved in Lipid and Glucose Metabolism. To further analyze the effects of drug treatment, we measured the messenger $(\mathrm{m}) \mathrm{RNA}$ levels of several genes known to be associated with the development of hepatic steatosis. Total RNA was isolated from the livers of ob/ob mice plus or minus treatment with Genz-123346 for 7 weeks and quantitative RT-PCR was performed. SREBP-1c, a key transcription factor involved in fatty acid and triglyceride synthesis, was upregulated in ob/ob livers compared to leans, as reported ${ }^{14}$ (Fig. 3). Genz-123346 treatment resulted in a trend toward decreased mRNA levels of SREBP-1c and several genes involved in fatty acid synthesis, including ATP citrate lyase (ACL), acyl-CoA carboxylase-1 (ACC1), fatty acid synthase (FAS), and stearoylCoA desaturase-1 (SCD-1). Genz-123346 did not appear to affect the expression of genes involved in mitochondrial and peroxisomal $\beta$-oxidation, such as peroxisome proliferator-activated receptor $\alpha$ (PPAR- $\alpha$ ) or acyl-CoA oxidase (AOX). The mRNA levels of uncoupling protein 2 (UCP2) were up-regulated in ob/ob mice compared to leans as reported, ${ }^{15}$ but not significantly altered in the drug-treated livers (Fig. 3). Likewise, mRNA levels of PPAR- $\gamma$ were highly elevated in ob/ob mice, consistent with what has been reported, ${ }^{16}$ but were not lowered in response to drug treatment.

In addition, we analyzed the mRNA levels of the gluconeogenic gene glucose-6-phosphatase (G6Pase), which was elevated in ob/ob mice and significantly reduced in the drug-treated animals (Fig. 3). The mRNA levels of the inflammatory cytokine tumor necrosis factor- $\alpha$ (TNF- $\alpha$ ) were also elevated nearly three-fold in the ob/ob mouse livers, and drug treatment reduced liver TNF- $\alpha$ mRNA levels to that found in lean animals. Lastly, we observed elevated levels of liver procollagen $\alpha$ type I (PRO- $\alpha 1$ ) mRNA in ob/ob mice, even though ob/ob mice do not develop overt fibrosis. Interestingly, the mRNA levels of procollagen I were reduced in the livers of animals treated with Genz-123346 (Fig. 3). Overall, the data indicate that drug treatment dampened a broad spectrum of genes associated with the development of hepatic steatosis, including genes involved in lipogenesis, gluconeogenesis, and inflammation. These concerted changes in gene expression are consistent with the observed decrease in steatosis and markers of liver pathology.

Genz-123346 Inhibited the Development of $\mathrm{He}$ patic Steatosis in DIO Mice. A second, less severe, and more slowly progressing rodent model of hepatic steatosis is that of C57BL/6 mice placed on a high-fat diet for several weeks. We previously showed that treating DIO 
Fig. 3. Effect of Genz-123346 on the expression of lipogenic, gluconeogenic, fibrotic, and inflammatory genes in the liver. Mice were treated with Genz-123346 for 7 weeks $(60 \mathrm{mg} / \mathrm{kg}$ twice daily oral gavage $=120 \mathrm{mg} / \mathrm{kg} /$ day). At the end of treatment, the mice were fasted overnight and then refed for 3 hours prior to harvesting the liver. Total liver RNA was isolated and quantitative RT-PCR was performed. SREBP-1c, sterol regulatory element binding protein-1c; ACL1, ATP citrate lyase 1; ACC1, acyl-CoA carboxylase-1; FAS, fatty acid synthase; SCD-1, stearoyl-CoA desaturase-1; PPAR- $\alpha$, peroxisome proliferator-activated receptor $\alpha$; AOX, acyl-CoA oxidase; UCP2, uncoupling protein 2 ; PPAR- $\gamma$, peroxisome proliferatoractivated receptor $\gamma$; TNF- $\alpha$, tumor necrosis factor $\alpha$; PRO- $\alpha 1$, procollagen a type 1; G6Pase, glucose-6phosphatase. Data shown as mean \pm SEM $(n=3-4$ mice per group). ${ }^{*} p<0.05,{ }^{* *} p<0.01$ Genz-123346 ob/ob versus water ob/ob.
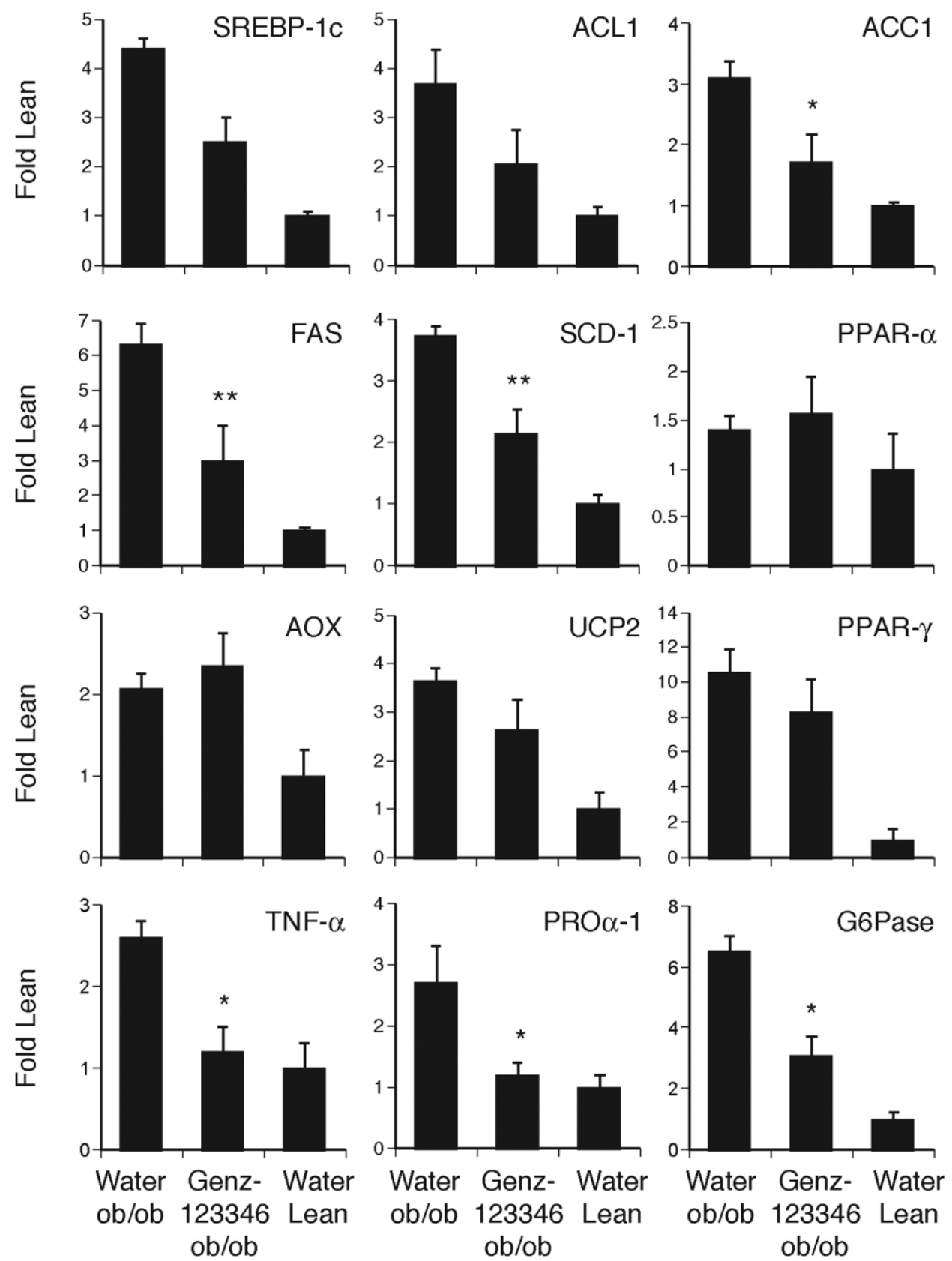

mice with Genz-123346 increased insulin sensitivity, improved glucose tolerance, and lowered HbA1c levels. ${ }^{11}$ To further examine the effects of inhibiting GSL synthesis on liver pathology in this model, we initiated treatment after the mice had been placed on a high-fat diet ( $45 \% \mathrm{kcal}$ fat) for 8 weeks. Genz-123346 or placebo (water) was administered to the DIO mice by daily oral gavage $(125 \mathrm{mg} / \mathrm{kg} /$ day) for 20 weeks. At the end of the treatment period, the fat and lean masses of the livers were determined (see Materials and Methods). The livers of animals treated with Genz-123346 exhibited a significantly reduced ratio of fat mass to lean mass compared to the livers of animals treated with placebo (water) (Fig. 4A). Serum ALT levels were also measured, and in the placebo-treated group serum ALT levels increased markedly over time (Fig. 4B). In contrast, in the drug-treated group the increases in ALT levels were significantly abated.

Prior to the initiation of treatment only a few, widely dispersed lipid vacuoles were visible in the stained liver sections, even though the animals had received the highfat diet for 8 weeks (Fig. 4C). However, after 12 and 20 additional weeks on the high-fat diet the number and size of the lipid vacuoles increased considerably in the placebo (water)-treated animals. In contrast, in the animals treated with Genz-123346 (125 mg/kg daily oral gavage), the livers contained significantly fewer and smaller-sized 

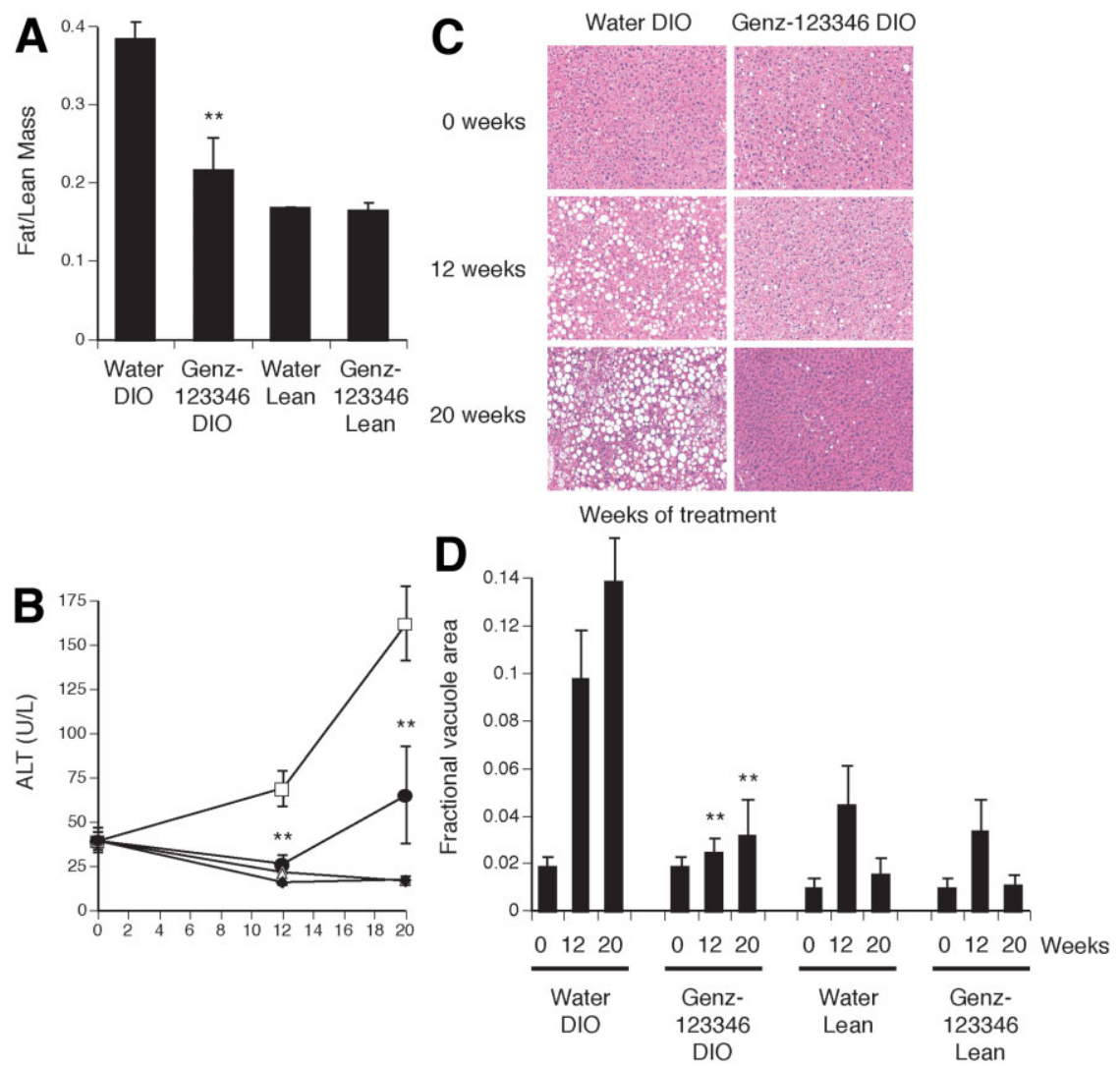

Fig. 4. Genz-123346 reduced the increase in liver fat mass, ALT levels, and hepatic steatosis in DIO mice. Mice were placed on a high-fat diet for 8 weeks. At the end of this initial period, one group of mice was sacrificed and the livers harvested ( 0 weeks timepoint). The remaining mice were then treated with placebo (water) or Genz-123346 (125 mg/ kg/ day) by daily oral gavage for 12 or 20 weeks, while still on the high-fat diet. At the end of the treatment the livers were harvested and the amount of fat and lean mass quantitated by magnetic resonance imaging. (A) Ratio of fat to lean mass in the liver at 20 weeks. (B) Serum ALT levels. Open squares, Water DI0; filled circles, Genz-123346 DI0; open triangles, Water Lean; filled diamonds, Genz-123346 Lean. (C) Liver sections stained with hematoxylin and eosin. (D) Morphometric analysis. Data shown as mean \pm SEM ( $n=6-16$ mice per group). $* P<0.05, * * P<0.01$ Genz-123346 DI0 versus water DIO. vacuoles compared to the water-treated group (Fig. 4C). Morphometric analysis confirmed this reduction in fractional lipid vacuole area from 0.098 (placebo) to 0.025 (Genz-123346) at 12 weeks, representing a 74\% decrease, and from 0.139 (placebo) to 0.032 (Genz-123346) at 20 weeks, a 77\% decrease (Fig. 4D). Thus, inhibiting GSL synthesis almost completely prevented the development of hepatic steatosis in DIO mice, along with decreasing the associated pathological marker ALT.

Genz-123346 Partially Reversed Established Steatosis in Aged DIO Mice. To determine if inhibiting GSL synthesis could reverse preexisting, well-established hepatic steatosis, we treated older DIO mice that had been placed on a high-fat diet ( $45 \%$ of kcal from fat) for 31 weeks. The mice were treated with placebo (water) or Genz-123346 (125 mg/kg) by daily oral gavage for 17 weeks. At the end of the treatment period the livers were harvested and the fat and lean masses of the livers measured. As was observed in the younger DIO mice, the livers of the older DIO animals treated with Genz123346 exhibited a significantly reduced ratio of fat mass to lean mass compared to the livers of animals treated with placebo (water) (Fig. 5A). ALT levels were elevated in the DIO mice compared to the lean animals prior to the start of treatment. Genz-123346 treatment decreased the levels of ALT, indicating a reversal of liver pathology (Fig.
5B). Histological analysis showed that these mice exhibited extensive lipid deposition throughout the liver prior to the initiation of drug treatment, with numerous lipidfilled vacuoles and foamy cells (Fig. 5C). The livers of the placebo (water)-treated control animals continued to exhibit pervasive steatosis to a degree similar to that observed prior to the start of treatment. In contrast, in the animals treated with Genz-123346 the livers contained fewer vacuoles and cells having a foamy appearance (Fig. 5C). From morphometric analysis a high degree of variability in the fractional vacuole area was observed among individual animals, even in the lean animals (Fig. 5D). The reduction in vacuole area due to drug treatment did not reach statistical significance. Nevertheless, in the majority of animals treated with Genz-12336 the fractional vacuole area was reduced compared to that observed in the water-treated DIO mice.

\section{Discussion}

Recent studies have demonstrated improvements in insulin sensitivity and glucose homeostasis in animal models of type 2 diabetes using inhibitors of glucosylceramide synthase, the enzyme involved in the first step of GSL synthesis. Here we further examined the specific effects of this inhibitor on liver morphology and pathology 

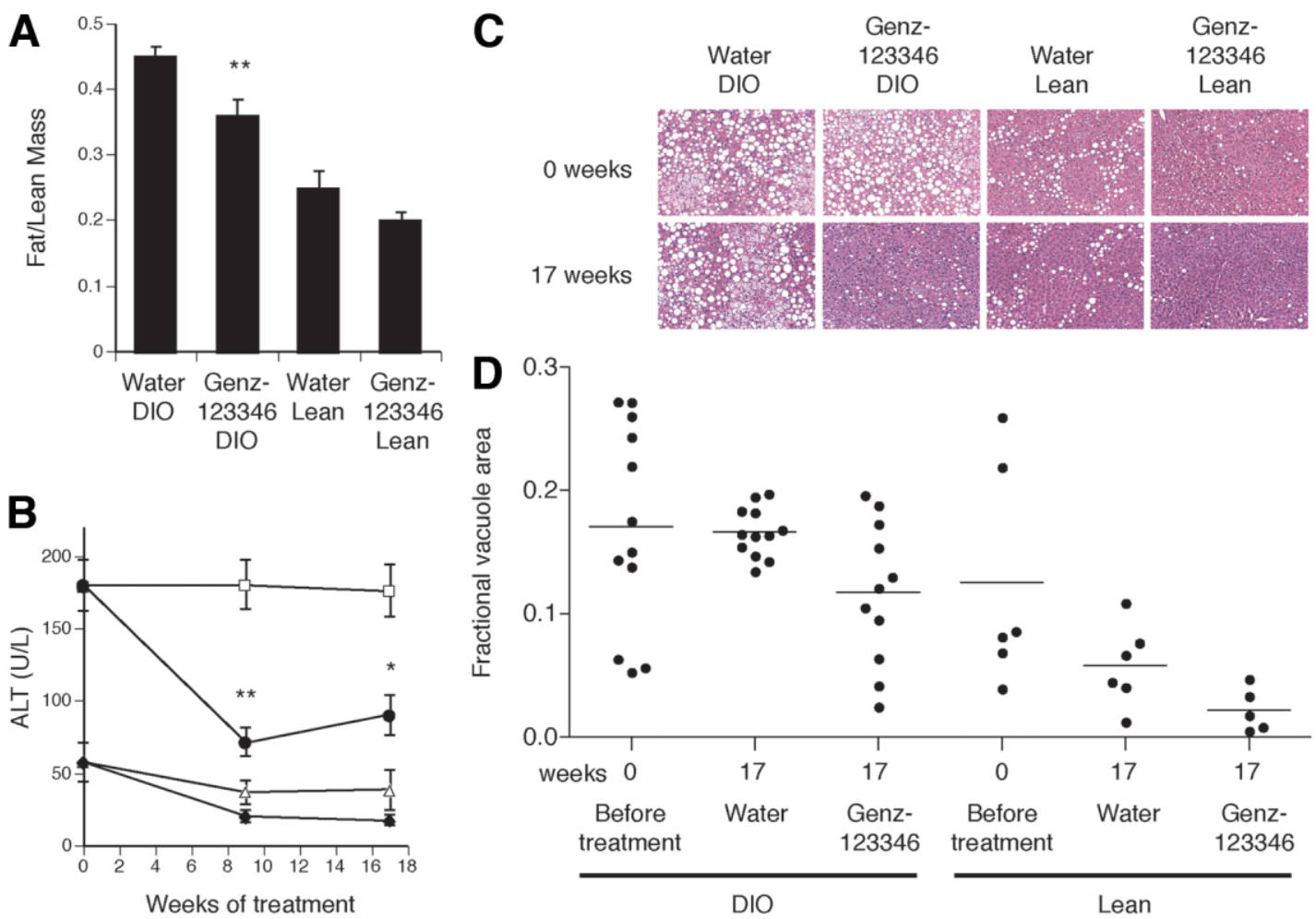

Fig. 5. Genz-123346 decreased liver fat mass, ALT levels, and partially reversed established steatosis in aged DI0 mice. Mice were placed on a high-fat diet for 31 weeks. At the end of this initial period, one group of mice was sacrificed and the livers harvested ( 0 weeks timepoint). The remaining mice were then treated with Genz-123346 (125 mg/kg/day) or placebo (water) by daily oral gavage for 17 weeks, while still on the high-fat diet. During this period blood was collected by eyebleed at 9 weeks for ALT levels. At the end of the treatment the livers were harvested and the amount of fat and lean mass quantitated by magnetic resonance imaging. (A) Ratio of fat to lean mass in the liver. (B) Serum ALT levels. Open squares, Water DI0; filled circles, Genz-123346 DI0; open triangles, Water Lean; filled diamonds, Genz-123346 Lean. (C) Liver sections stained with hematoxylin and eosin. (D) Morphometric analysis. Data shown as mean \pm SEM ( $n=5-12$ mice per group). $* P<0.05, * * P<0.01$ Genz-123346 DIO versus water DIO.

in ob/ob and DIO mice. The ob/ob mice rapidly develop severe hepatic steatosis and IR. Compared to placebotreated mice, we showed that treatment of ob/ob mice with the glucosylceramide synthase inhibitor Genz123346 reduced steatosis in the liver and decreased several markers of liver pathology and inflammation. Interestingly, although ob/ob mice are not considered a model of $\mathrm{NASH}$, drug treatment decreased the expression of the inflammatory cytokine TNF- $\alpha$, which is associated with the development of NASH. ${ }^{17}$ In addition, inhibiting GSL synthesis not only prevented hepatic steatosis in the DIO mouse model, but also partially reversed established steatosis.

The basis for the reduction in steatosis in the liver is unclear, although the simplest explanation is an increase in insulin sensitivity in one or more tissues that arise from the previously reported interaction of GSLs, in particular GM3, with the insulin receptor. ${ }^{8-10,18}$ It was initially shown in 3T3-L1 adipocytes that exogenously added GM3 inhibited insulin signaling, and conversely lowering GM3 levels enhanced signaling. ${ }^{9}$ Although the ability of Genz-123346 to increase insulin signaling in the liver remains to be deter- mined, we have previously observed increased insulin receptor and mammalian target of rapamycin (mTOR) phosphorylation in the ob/ob mouse liver using a different glucosylceramide synthase inhibitor. ${ }^{12}$ However, so far we have not been able to consistently observe elevated levels of GM3 or other GSLs in ob/ob livers compared to levels in lean animals. We therefore speculate that disease-related alterations in GSLs and the major effects of Genz-123346 could take place in other tissues such as adipose and muscle. GM3 synthase has been shown to be elevated in adipose tissue from ob/ob mice and Zucker rats, ${ }^{9}$ and we are currently investigating which GSLs are upregulated in this tissue. Visceral fat has an important role in the regulation of hepatic triglyceride content as a source of not only FFA but also the hormones adiponectin, resistin, leptin, etc., and several proinflammatory cytokines. ${ }^{19}$ IR in muscle and subsequent increases in circulating glucose levels also contributes to the development of hepatic steatosis, and we have previously demonstrated markedly increased insulin receptor and Akt activation in muscle of DIO mice treated with Genz123346. ${ }^{11}$ Thus, the observed decrease in liver mRNA levels of several lipogenic genes and other genes associated with 
steatosis could possibly be explained as an indirect consequence of an overall increase in insulin sensitivity in liver, adipose, and muscle. GSL synthesis inhibition did appear to reduce steatosis to a greater degree in the DIO mouse compared to ob/ob mice, correlating with the relative severity of IR in the two models.

An improvement in insulin signaling does not preclude possible direct effects of Genz-123346 on steatosis. GSLs have been shown to modulate the activity of not only the insulin receptor but also other membrane-associated kinases and receptors, e.g., growth factor and cytokine receptors. ${ }^{20}$ Conceivably, GSL-mediated inhibition of cytokine and immune cell signaling would decrease inflammation, an important contributing factor to the development of steatosis and NASH. ${ }^{21}$ Also, drugs that increase insulin sensitivity do not always decrease hepatic lipid accumulation, a prominent example being the PPAR- $\gamma$ agonist rosiglitazone. In ob/ob and DIO mice rosiglitazone effectively lowered blood glucose levels but increased liver steatosis. ${ }^{22}$ This phenomenon appears to be mouse-specific and is likely due to the elevated expression of the PPAR- $\gamma$ receptor in the ob/ob mouse compared to low levels in normal human livers. ${ }^{23}$ Nevertheless, the data show that increasing insulin sensitivity does not necessarily decrease steatosis. Also unlike rosiglitazone, treatment with Genz-123346 reduced the number of lipid vacuoles in other tissues of the DIO mouse, such as kidney (data not shown). This result is a desirable feature to treat not only NAFLD but also type 2 diabetes, as ectopic lipid accumulation is an early pathophysiological event. ${ }^{24}$

One concern with inhibiting GSL synthesis is the impact on ceramide metabolism. Theoretically, ceramide may increase as a result of inhibiting glucosylceramide synthase. However, we have not observed any increase in ceramide levels in plasma or liver as a result of drug treatment (see Supporting Material and Ref. 12). One possible reason is that there remain several other pathways for ceramide conversion, e.g., ceramide to sphingosine, sphingomyelin, galactosylceramide, or ceramide 1-phosphate. So far we have not observed any increase in sphingomyelin, sphingosine, or sphingosine 1-phosphate in plasma from normal or diabetic rats treated with GSL inhibitors (data not shown). Nevertheless, the long-term consequences of altering the flux of sphingolipid metabolism remain to be determined.

Currently there is no generally accepted treatment for fatty liver disease and NASH. Metformin and thiazolidinediones have shown some benefit in clinical trials, with improvements in IR markers, liver function tests, and positive histological changes in some cases. ${ }^{25-27}$ However, the small sample size and lack of a placebo arm in many of the studies precludes definitive conclusions for these drugs in treating fatty liver disease. Inhibiting key transcription factors or enzymes involved in lipogenesis, such as ChREBP, DGAT-2, or ACC1, can decrease hepatic steatosis and in turn improve IR. ${ }^{28-30}$ GLP-1 agonists are also potential treatments. ${ }^{31}$ Given the multifactorial nature of hepatic steatosis and NASH a combination of therapies will likely be required to fully treat these conditions. Lowering GSLs represents another novel and highly promising approach to address several of the important underlying pathologies of fatty liver disease.

Acknowledgment: We thank WeiLien Chuang, Leocadia Mosquea, Ronald Scheule, Joanne Donovan, and the Departments of Pathology and Comparative Medicine staff for their advice and technical support.

\section{References}

1. Adams LA, Angulo P, Lindor KD. Nonalcoholic fatty liver disease. CMAJ 2005;172:899-905.

2. Perlemuter G, Bigorgne A, Cassard-Doulcier AM, Naveau S. Nonalcoholic fatty liver disease: from pathogenesis to patient care. Nat Clin Pract Endocrinol Metab 2007;3:458-469.

3. Szczepaniak LS, Nurenberg P, Leonard D, Browning JD, Reingold JS, Grundy S, et al. Magnetic resonance spectroscopy to measure hepatic triglyceride content: prevalence of hepatic steatosis in the general population. Am J Physiol Endocrinol Metab 2005;288:E462-E468.

4. Dunn W, Schwimmer JB. The obesity epidemic and nonalcoholic fatty liver disease in children. Curr Gastroenterol Rep 2008;10:67-72.

5. Browning JD, Horton JD. Molecular mediators of hepatic steatosis and liver injury. J Clin Invest 2004;114:147-152.

6. Abdelmalek MF, Diehl AM. Nonalcoholic fatty liver disease as a complication of insulin resistance. Med Clin North Am 2007;91:1125-1149, ix.

7. Donnelly KL, Smith CI, Schwarzenberg SJ, Jessurun J, Boldt MD, Parks EJ. Sources of fatty acids stored in liver and secreted via lipoproteins in patients with nonalcoholic fatty liver disease. J Clin Invest 2005;115:13431351.

8. Nojiri H, Stroud M, Hakomori S. A specific type of ganglioside as a modulator of insulin-dependent cell growth and insulin receptor tyrosine kinase activity. Possible association of ganglioside-induced inhibition of insulin receptor function and monocytic differentiation induction in HL-60 cells. J Biol Chem 1991;266:4531-4537.

9. Tagami S, Inokuchi JJ, Kabayama K, Yoshimura H, Kitamura F, Uemura S, et al. Ganglioside GM3 participates in the pathological conditions of insulin resistance. J Biol Chem 2002;277:3085-3092.

10. Yamashita T, Hashiramoto A, Haluzik M, Mizukami H, Beck S, Norton A, et al. Enhanced insulin sensitivity in mice lacking ganglioside GM3. Proc Natl Acad Sci U S A 2003;100:3445-3449.

11. Zhao H, Przybylska M, Wu IH, Zhang J, Siegel C, Komarnitsky S, et al. Inhibiting glycosphingolipid synthesis improves glycemic control and insulin sensitivity in animal models of type 2 diabetes. Diabetes 2007;56: 1210-1218

12. Aerts JM, Ottenhoff R, Powlson AS, Grefhorst A, van Eijk M, Dubbelhuis $\mathrm{PF}$, et al. Pharmacological inhibition of glucosylceramide synthase enhances insulin sensitivity. Diabetes 2007;56:1341-1349.

13. McEachern KA, Fung J, Komarnitsky S, Siegel CS, Chuang WL, Hutto E, et al. A specific and potent inhibitor of glucosylceramide synthase for substrate inhibition therapy of Gaucher disease. Mol Genet Metab 2007; 91:259-267.

14. Shimomura I, Bashmakov Y, Horton JD. Increased levels of nuclear SREBP-1c associated with fatty livers in two mouse models of diabetes mellitus. J Biol Chem 1999;274:30028-30032. 
15. Chavin KD, Yang S, Lin HZ, Chatham J, Chacko VP, Hoek JB, et al. Obesity induces expression of uncoupling protein-2 in hepatocytes and promotes liver ATP depletion. J Biol Chem 1999;274:5692-5700.

16. Memon RA, Hotamisligil GS, Wiesbrock SM, Uysal KT, Faggioni R, Moser AH, et al. Up-regulation of peroxisome proliferator-activated receptors (PPAR-alpha) and PPAR-gamma messenger ribonucleic acid expression in the liver in murine obesity: troglitazone induces expression of PPAR-gamma-responsive adipose tissue-specific genes in the liver of obese diabetic mice. Endocrinology 2000;141:4021-4031.

17. Diehl AM. Lessons from animal models of NASH. Hepatol Res 2005;33: 138-144.

18. Kabayama K, Sato T, Kitamura F, Uemura S, Kang BW, Igarashi Y, et al. TNFalpha-induced insulin resistance in adipocytes as a membrane microdomain disorder: involvement of ganglioside GM3. Glycobiology 2005;15:21-29.

19. Shoelson SE, Lee J, Goldfine AB. Inflammation and insulin resistance. J Clin Invest 2006;116:1793-1801.

20. Miljan EA, Bremer EG. Regulation of growth factor receptors by gangliosides. Sci STKE 2002:RE15.

21. Carter-Kent C, Zein NN, Feldstein AE. Cytokines in the pathogenesis of fatty liver and disease progression to steatohepatitis: implications for treatment. Am J Gastroenterol 2008;103:1036-1042.

22. Garcia-Ruiz I, Rodriguez-Juan C, Diaz-Sanjuan T, Martinez MA, MunozYague T, Solis-Herruzo JA. Effects of rosiglitazone on the liver histology and mitochondrial function in ob/ob mice. HePATOLOGY 2007;46:414-423.

23. Caldwell SH, Argo CK. Divergent effects of peroxisome proliferator-activated receptor-gamma ligands in human and mouse nonalcoholic steatohepatitis. Hepatology 2007;46:285-287.
24. Unger RH. Minireview: weapons of lean body mass destruction: the role of ectopic lipids in the metabolic syndrome. Endocrinology 2003;144:51595165 .

25. de Oliveira CP, Stefano JT, de Siqueira ER, Silva LS, de Campos Mazo DF, Lima VM, et al. Combination of $\mathrm{N}$-acetylcysteine and metformin improves histological steatosis and fibrosis in patients with non-alcoholic steatohepatitis. Hepatol Res 2008;38:159-165.

26. Duseja A, Das A, Dhiman RK, Chawla YK, Thumburu KT, Bhadada S, et al. Metformin is effective in achieving biochemical response in patients with nonalcoholic fatty liver disease (NAFLD) not responding to lifestyle interventions. Ann Hepatol 2007;6:222-226.

27. Lang L. Pioglitazone trial for NASH: results show promise. Gastroenterology 2007;132:836-838.

28. Dentin R, Benhamed F, Hainault I, Fauveau V, Foufelle F, Dyck JR, et al. Liver-specific inhibition of ChREBP improves hepatic steatosis and insulin resistance in ob/ob mice. Diabetes 2006;55:2159-2170.

29. Choi CS, Savage DB, Kulkarni A, Yu XX, Liu ZX, Morino K, et al. Suppression of diacylglycerol acyltransferase-2 (DGAT2), but not DGAT1, with antisense oligonucleotides reverses diet-induced hepatic steatosis and insulin resistance. J Biol Chem 2007;282:22678-22688.

30. Savage DB, Choi CS, Samuel VT, Liu ZX, Zhang D, Wang A, et al. Reversal of diet-induced hepatic steatosis and hepatic insulin resistance by antisense oligonucleotide inhibitors of acetyl-CoA carboxylases 1 and 2 . J Clin Invest 2006;116:817-824.

31. Ding X, Saxena NK, Lin S, Gupta NA, Anania FA. Exendin-4, a glucagonlike protein-1 (GLP-1) receptor agonist, reverses hepatic steatosis in ob/ob mice. Hepatology 2006;43:173-181. 